\title{
The effects of embryo competition with mixed mating on the genetic load in plants
}

\author{
R. G. LATTA \\ Department of EPO Biology, University of Colorado, Boulder, CO 80309, U.S.A.
}

\begin{abstract}
Many plant species abort a large number of seeds, often producing an increase in the fitness of progeny, and a decrease in the proportion of inbred offspring. Where genetic load is caused by deleterious recessive mutations in mutation-selection balance, the removal of inbred offspring may prevent the expression of mutations as homozygotes. This would shelter the mutations from selection, and permit an increase in the genetic load relative to the case with no competition among embryos. This study employs existing models of genetic load to assess the effects of embryo competition on load. Mutations which are not expressed in the embryo (e.g. chlorophyll deficiency mutations) are sheltered from selection by the removal of inbred seed, and increase in frequency relative to the case without embryo competition. Moreover, if embryo competition is discontinued after several generations, expression of the accumulated load causes a temporary, but pronounced drop in mean fitness. By contrast, mutations which are expressed in the embryo may affect the outcome of competition, and thus experience added selection relative to the case with no competition. Genetic load at these loci may thus be reduced, although the exact outcome depends upon the expression of the mutations in heterozygotes. The mechanism of embryo competition must be known before the long-term effects of management practices which enhance embryo competition can be predicted. Even where selective abortion increases the fitness of stocks over the long term, the accumulation of additional mutations can produce a dramatic loss of fitness if selective seed abortion is discontinued. Thus, once a programme to enhance selective abortion is begun, it may be impossible to cease this programme without irreparable damage to the stocks.
\end{abstract}

Keywords: embryo competition, inbreeding depression, mutation, purging, supplemental mass-pollination.

\section{Introduction}

There is a trade-off between the expression of deleterious recessive mutations, and the accumulation of those mutations when their expression is masked. Although close inbreeding generally leads to reduced vigour of progeny (inbreeding depression), systematic inbreeding can reduce the amount of inbreeding depression by exposing recessive mutations to selection (Lande \& Schemske, 1985; D. Charlesworth et al., 1990). Empirically, populations practising a lower level of inbreeding tend to have greater levels of inbreeding depression (Holtsford \& Ellstrand, 1990; Latta \& Ritland, 1994). Thus the immediate fitness effects of a particular mating strategy may be at odds with longer term success of the lineage. This fact has important implications for the management of captive or domestic stocks.

The elimination of fertilized ovules prior to ripening seems to occur in many species of plants, and is most common in longer-lived shrubs and trees (Sorensen, 1982; Bawa \& Webb, 1984; Wiens, 1984). There are several hypotheses to explain seed abortion, including resource limitation, inviability of some zygotes and the competitive elimination of less fit zygotes from the progeny (Gorchov \& Estabrook, 1987; Herrera, 1990). Outcrossing rates measured at seed maturity (secondary outcrossing rate) are frequently higher than those at fertilization, especially in species with high genetic load, such as the conifers (Sorensen, 1982; Karkainen \& Savolainen, 1992). This suggests the elimination of selfed embryos during seed development, which could result in part from simple inviability of inbred zygotes. However, seed abortion is often nonrandom among fruits, such that survival of one embryo is associated with the death of others (Caspar, 1988; Herrera, 1990). This suggests that seed abortion is a function of competition among embryos, as opposed to postzygotic self incompatibility, or inviability of 
selfed seeds, which are presumably independent of other embryos in the fruit. For example, in conifers, the presence of more than one egg in an ovule allows for two embryos potentially to compete for presence in the seed (Sorensen, 1982), provided that both are viable. This phenomenon, known as simple polyembryony, may serve to ensure that the maternal tree invests its resources in the most fit embryos (Haig, 1992). Similar processes are conceivable for angiosperms, with ovules competing within a fruit, as in Cryptantha flava (Caspar, 1988).

Enhancing this competition could allow the plant (or plant breeder) to create a higher mean fitness in the progeny. Techniques such as supplemental masspollination (Daniels, 1978; Crook \& Friedman, 1992)mayservetoincreasethenumberofzygotesformed, and thus increase the range of embryo genotypes from which the plant could select. However, elimination of inbred zygotes may also protect mutations from selection, and thereby increase the genetic load over the long term (Klekowski, 1982; Haig, 1992). The purpose of this paper is to model the purging of recessive deleterious mutations under several mechanisms of embryo competition. The models are simply an application of existing theory which describes the dynamics of inbreeding depression caused by recessive deleterious mutations (Kondrashov, 1985; D. Charlesworth et al., 1990). I investigate the case where embryo competition is determined by the genotype at loci carrying mutations, as well as the case in which mutations are not expressed in the embryo and therefore do not influence the outcome of competition.

\section{The model}

I examined a multilocus model of genetic load using the approach outlined by Kondrashov (1985) and D. Charlesworth et al. (1990). Inbreeding depression is assumed to be caused by recessive deleterious mutations, which are maintained at low frequency by recurrent mutation. An infinite number of potentially mutable loci is assumed, and the population is modelled by tracking the proportion of individuals heterozygous for $i$ mutations and homozygous for $j$ mutations, denoted $P_{i j}$. The model assumes an infinite population of hermaphrodites with nonoverlapping generations, and no biparental inbreeding.

There are three stages to the Kondrashov model: mutation, mating and selection. Mutation increases the number of mutations an individual carries as heterozygotes, while selection reduces the frequency of genotypes with high numbers of mutations. What follows is a brief description of the model; for details on the derivation of these recursions see D. Charlesworth et al. (1990). For the mutation rate, $U$, per genome, the probability of $0,1,2 \ldots$ mutations occurring in an individual can be calculated, and then summed across all genotypes. Genotype frequencies after mutation are thus:

$P_{i j}^{\prime}=\mathrm{e}^{-U} \sum_{n=0}^{i} P_{n j} U^{i-n} /(i-n) !$.

Genotypes then undergo mating according to the rules of Mendelian segregation, with a proportion $t$ created through outcrossing, and $1-t$ created by selfing. For outcrossing, the array of possible gametes is calculated as

$f_{i}=\sum_{m=0}^{i} \sum_{n=i-m}^{\infty} P^{\prime}{ }_{n m} 0.5^{n} n ! /(i-m) !(n+m-i) !$.

The gametes are then combined at random assuming that all mutations are sufficiently rare that they are never made homozygous through outcrossing:

$P^{\prime \prime}(x) i 0=\sum_{n=0}^{i} f_{n} f_{i-n}$

Genotype frequencies among selfed offspring are:

$P^{\prime \prime}{ }_{(s) i j}=\sum_{n} \sum_{m} P^{\prime}{ }_{n m} T_{i j n m}$,

where $T_{i j n m}$ is the probability of an individual carrying $n$ and $m$ mutations giving rise to a selfed offspring with $i$ and $j$ mutations as defined by Kondrashov (1985, p. 640).

Selection on the mature plants is imposed by assigning a fitness loss of $s$ for each homozygous mutation, and $h s$ for each heterozygous mutation (for $h$, the proportional expression of the mutation in heterozygotes). Fitness interactions are multiplicative across loci, and it is assumed throughout that mutations are sufficiently rare that mean fitness of selfs is greater than zero (see Lande et al., 1994 for the relaxation of this assumption). Thus the fitness of an individual with $i$ and $j$ mutations is:

$w_{i j}=(1-h s)^{i} \times(1-s)^{j}$.

I investigated two sets of parameters, one to model the effects of mildly deleterious, partly recessive mutations $(s=0.1, h=0.2)$, and the other to model the effects of severe mutations (lethal or sublethal: $s=0.9, h=0.02$ ). Per genome, mutation rates, $U$, took values from 0.05 to 0.5 . Because $\mathrm{B}$. Charlesworth et al. (1990) estimate $U$ as 'at least 0.5 , these values may be low for natural populations, but serve to give the qualitative pattern of results without excessive computer time.

I assumed that embryo competition occurs in 
pairs, with one seed surviving and the other being aborted. Although any number of seeds might compete with an arbitrary number surviving, competition in pairs is most mathematically tractable, and will suffice to determine the qualitative effects upon load. For each maternal genotype, I calculated the array of possible progeny genotypes. Embryos were then paired at random, such that they shared either their mother (ovules competing within a fruit-the 'angiosperm model'), or the same maternal gamete (embryos competing within an ovule-the 'polyembryony model'). A zygote with genotype ij will be paired with zygote $\mathrm{nm}$ with a probability equal to the frequency of $\mathrm{nm}$ genotypes in the progeny array of that maternal plant. Embryos win the competition with a probability proportional to their relative fitnesses. Thus

$P^{\prime \prime \prime}{ }_{i j}=2 \times P^{\prime \prime}{ }_{i j} \times \sum_{n} \sum_{m}\left[P_{n m} \times w_{i j} /\left(w_{i j}+w_{n m}\right)\right]$.

For mutations not expressed in the embryo (e.g. chlorophyll deficiency mutations), elimination of lower fitness selfed zygotes will be independent of genotype. To model this case, paired embryos won the competition dependent solely upon the 'matingtype' (self or outcross) of the two embryos involved. Where outcrossed embryos competed with selfed embryos, selfed embroys were at a disadvantage and were preferentially eliminated with a probability proportional to the relative fitness of selfed zygotes.

$$
\begin{aligned}
& P^{\prime \prime \prime}{ }_{(x) i j}=2 \times P^{\prime \prime}{ }_{(x) i j} \times\left[t / 2+(1-t) \times w_{\mathrm{x}} /\left(w_{\mathrm{x}}+w_{\mathrm{s}}\right)\right] \\
& P^{\prime \prime \prime}{ }_{(s) i j}=2 \times P^{\prime \prime}{ }_{(s) i j} \times\left[(1-t) / 2+t \times w_{\mathrm{s}} /\left(w_{\mathrm{x}}+w_{\mathrm{s}}\right)\right],
\end{aligned}
$$

where $w_{\mathrm{x}}$ and $w_{\mathrm{s}}$ are the fitnesses of outcrossed and selfed embryos, respectively. Because the matingtype model assumes that load loci are not expressed in the embryo, $w_{\mathrm{x}}$ and $w_{\mathrm{s}}$ are arbitrarily defined, rather than calculated from the genotype at load loci. Selection on load loci does occur in adult plants, however, such that the characteristics of the mutations (mild vs. severe) will affect their evolutionary dynamics.

For each set of parameters describing the outcrossing rate and load, the model was run for 100 generations until the mutations reached a mutationselection equilibrium. At this point, embryo competition was introduced as described above. The frequency of mutations, the inbreeding depression and the mean fitness of the mature seeds were recorded each generation, along with the secondary outcrossing rate (the proportion of outcrossed seeds occurring among the nonaborted seed). Once these parameters reached equilibrium, the selective seed abortion was discontinued to determine the consequences of a pollinator failure, or other reduced fertilization rate, which would reduce the degree of embryo competition.

\section{Results and discussion}

In each model, competition among embryos caused an increase in the proportion of outcrossed seed between fertilization and seed maturity (Table 1). Also, there was an initial increase in mean progeny fitness with embryo competition regardless of the mechanism (Fig. 1). In many (but by no means all) cases, this initial advantage eroded as the relaxed selective environment allowed mutations to build up to higher levels than were originally present. The build-up of genetic load and erosion of progeny fitness was most pronounced for the mating-type model where the outcome of embryo competition was not determined by the genotype of the seeds, but rather by their inbreeding coefficient. For this model, the reduction in fitness over the long term was greater than the initial fitness advantage caused by the elimination of less fit embryos. The change in equilibrium load with removal of selfed zygotes was exactly predicted by the change in the secondary outcrossing rate. When embryo competition was discontinued, and mutations were no longer sheltered from selection, the expression of the accumulated load created a pronounced fitness loss in the population (Fig. 1). As the accumulated load was eliminated, mean fitness rose again towards its original level.

When load loci are expressed in the embryo and form the basis for embryo competition (angiosperm and polyembryony models), the outcome of competition depended in a complex way upon the characteristics of the mutations and the outcrossing rate. Mildly deleterious mutations were not sheltered from selection, but rather selection against mutant alleles was reinforced by embryo competition. Because load did not increase, there was no erosion of the fitness gains attributable to elimination of less fit zygotes (Fig. 1a). Instead, mean fitness increased and load decreased, each moving asymptotically towards their new equilibria, returning to their original values when seed abortion was discontinued. By contrast, severe mutations accumulated with embryo competition in the polyembryony model, and at intermediate outcrossing rates in the angiosperm model (Fig. 1b), but, surprisingly, these same mutations decreased in frequency with embryo competition at high or low outcrossing rates in the angiosperm model (Table 1). In all cases with severe 
Table 1 Effects of embryo competition on mean fitness, genetic load (mean number of mutations per individual), inbreeding depression of adults $(\delta)$ and secondary outcrossing rate $\left(t_{s}\right)$

\begin{tabular}{|c|c|c|c|c|c|}
\hline & & Fitness & $\begin{array}{l}\text { No. of } \\
\text { mutations }\end{array}$ & $\delta$ & $t_{\mathrm{s}}$ \\
\hline \multicolumn{6}{|l|}{ Mild mutations } \\
\hline \multirow{2}{*}{\multicolumn{6}{|c|}{$\begin{array}{l}\text { Angiosperms } \\
t=0.9\end{array}$}} \\
\hline & $\begin{array}{l}\text { A } \\
\text { B }\end{array}$ & $\begin{array}{l}0.9561 \\
0.9566\end{array}$ & $\begin{array}{l}2.084 \\
2.048\end{array}$ & $\begin{array}{l}0.0322 \\
0.0296\end{array}$ & $\begin{array}{l}0.9000 \\
0.9016\end{array}$ \\
\hline & $\mathrm{C}$ & 0.9671 & $\begin{array}{l}2.040 \\
1.558\end{array}$ & 0.0226 & $\begin{array}{l}0.9010 \\
0.9012\end{array}$ \\
\hline & $\mathrm{D}$ & 0.9665 & 1.583 & 0.0245 & 0.9000 \\
\hline \multirow[t]{4}{*}{$t=0.5$} & A & 0.9679 & 1.102 & 0.0210 & 0.5000 \\
\hline & B & 0.9685 & 1.089 & 0.0196 & 0.5029 \\
\hline & C & 0.9740 & 0.9021 & 0.0164 & 0.5024 \\
\hline & D & 0.9731 & 0.9208 & 0.0175 & 0.5000 \\
\hline \multirow[t]{4}{*}{$t=0.1$} & A & 0.9738 & 0.6221 & 0.0155 & 0.1000 \\
\hline & B & 0.9740 & 0.6184 & 0.0152 & 0.1008 \\
\hline & C & 0.9754 & 0.5840 & 0.0146 & 0.1007 \\
\hline & D & 0.9751 & 0.5933 & 0.0148 & 0.1000 \\
\hline \multirow{2}{*}{\multicolumn{6}{|c|}{$\begin{array}{c}\text { Polyembryony } \\
t=0.5\end{array}$}} \\
\hline & A & 0.9679 & 1.102 & 0.0210 & 0.5000 \\
\hline & $\begin{array}{l}\mathrm{B} \\
\mathrm{C}\end{array}$ & $\begin{array}{l}0.9684 \\
0.9723\end{array}$ & $\begin{array}{l}0.9586 \\
0.9591\end{array}$ & $\begin{array}{l}0.0200 \\
0.0175\end{array}$ & $\begin{array}{l}0.5029 \\
0.5025\end{array}$ \\
\hline & $\mathrm{D}$ & 0.9717 & 0.9720 & 0.0185 & 0.5000 \\
\hline \multicolumn{6}{|l|}{ Mating-type } \\
\hline$t=0.5$ & A & 0.9679 & 1.102 & 0.0210 & 0.5000 \\
\hline \multirow[t]{3}{*}{$w_{\mathrm{s}} / w_{\mathrm{x}}=0.7$} & B & 0.9696 & 1.108 & 0.0204 & 0.5745 \\
\hline & C & 0.9667 & 1.219 & 0.0223 & 0.5747 \\
\hline & $\mathrm{D}$ & 0.9648 & 1.216 & 0.0230 & 0.5000 \\
\hline \multicolumn{6}{|c|}{ Severe mutations } \\
\hline \multicolumn{6}{|l|}{ Angiosperms } \\
\hline \multirow[t]{4}{*}{$t=0.9$} & A & 0.9666 & 0.8993 & 0.1767 & 0.9000 \\
\hline & B & 0.9810 & 0.8713 & 0.0347 & 0.9149 \\
\hline & C & 0.9811 & 0.8686 & 0.0344 & 0.9148 \\
\hline & D & 0.9666 & 0.8966 & 0.1762 & 0.9000 \\
\hline \multirow[t]{4}{*}{$t=0.5$} & A & 0.9738 & 0.2084 & 0.0450 & 0.5000 \\
\hline & B & 0.9898 & 0.1820 & 0.0130 & 0.5105 \\
\hline & C & 0.9885 & 0.2106 & 0.0510 & 0.5119 \\
\hline & D & 0.9706 & 0.2342 & 0.0504 & 0.5000 \\
\hline \multirow[t]{4}{*}{$t=0.1$} & A & 0.9748 & 0.1155 & 0.0258 & 0.1000 \\
\hline & B & 0.9884 & 0.0943 & 0.0104 & 0.1022 \\
\hline & $\mathrm{C}$ & 0.9873 & 0.1116 & 0.0112 & 0.1025 \\
\hline & D & 0.9730 & 0.1295 & 0.0288 & 0.1000 \\
\hline \multicolumn{6}{|l|}{ Polyembryony } \\
\hline$t=0.5$ & A & 0.9738 & 0.2084 & 0.0450 & 0.5000 \\
\hline & B & 0.9875 & 0.1922 & 0.0172 & 0.5105 \\
\hline & C & 0.9837 & 0.2573 & 0.0224 & 0.5138 \\
\hline & $\mathrm{D}$ & 0.9671 & 0.2630 & 0.0564 & 0.5000 \\
\hline \multicolumn{6}{|l|}{ Mating-type } \\
\hline \multirow{4}{*}{$w_{\mathrm{s}} / w_{\mathrm{x}}=0.7$} & A & 0.9738 & 0.2084 & 0.0450 & 0.5000 \\
\hline & B & 0.9766 & 0.2157 & 0.0463 & 0.5780 \\
\hline & C & 0.9734 & 0.2467 & 0.0528 & 0.5792 \\
\hline & D & 0.9702 & 0.2379 & 0.0512 & 0.5000 \\
\hline
\end{tabular}

Four values are reported for each run: (A), equilibrium values in the absence of embryo competition; (B), short term change in the first generation of embryo competition; (C), equilibrium with embryo competition; and (D), short-term effects of discontinuing embryo competition.

Mild mutations: $U=0.05, s=0.1, h=0.2$. Severe mutations: $U=0.05, s=0.9$, $h=0.02$.

$t$, Proportion of outcrossed embryos before competition; $w_{\mathrm{s}} / w_{\mathrm{x}}$, relative fitness of selfed embryos. 
mutations, the accumulation of genetic load did not outweigh the gains in fitness attributable to embryo competition so that fitness gains were maintained over the long term (Fig. 1b). Nonetheless, any accumulated load was expressed if embryo competition was discontinued, causing a sharp fitness loss, as in the mating type model.

The complex behaviour of the angiosperm and polyembryony models can be understood in terms of

a) Mild Mutations ( $s=0.1, h=0.2, U=0.15$ )

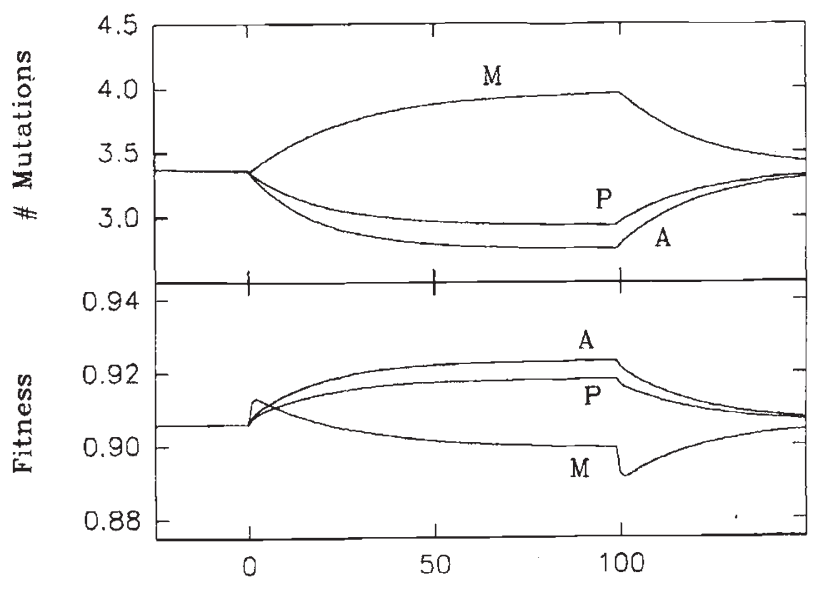

b) Severe Mutations ( $s=0.9, h=0.02, U=0.5$ )

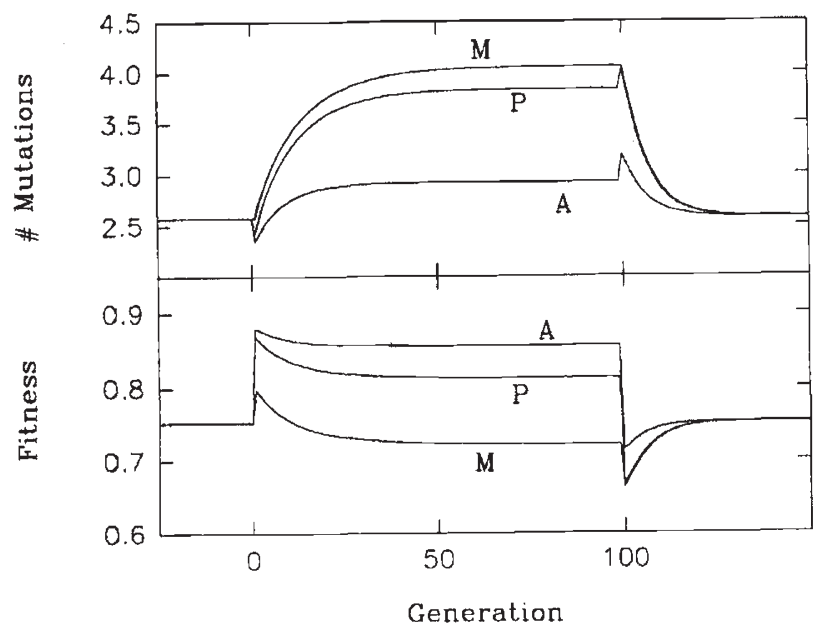

Fig. 1 Changes in genetic load and mean fitness over time with embryo competition. All graphs are for an outcrossing rate of 0.5 . (a) Mild mutations: $U=0.15, s=0.1$, $h=0.2$. (b) Severe mutations: $U=0.5, s=0.9, h=0.02$. Traces are given for each of the three possible mechanisms of embryo competition described in the text. Embryo competition was introduced to the model at generation zero and discontined at generation 100. A, angiosperm model; $\mathbf{P}$, polyembryony model; $\mathrm{M}$, mating-type model with relative fitness of selfed embryos $=0.7$. the dominance coefficient $h$. Mutations of mild effect generally have greater expression in the heterozygote than severe mutations $(h \approx 0.2-0.3$ for mild mutations; $h \approx 0.02$ for lethal and sublethal mutations: Simmons \& Crow, 1977). Thus for mild mutations, competition between embryos of genotype $A A$ and $A a$ (where $a$ denotes the mutant allele) will strongly favour the $A A$ homozygote. By contrast, if mutations are strongly recessive, $A A$ and $A a$ genotypes have similar fitness, and competition will not discriminate between them. Rather, competition will discriminate against the mutant homozygote in competition with the other two genotypes. Note that $A a$ vs. $a a$ competitions will favour the mutant allele because the heterozygote has an advantage. $A A$ vs. a a competition will tend to reduce the frequency of the mutant allele. In the polyembryony model, $A A$ vs. $a a$ pairings are impossible, because both embryos in a seed share the same maternal allele, and thus load increases with embryo competition (Fig. 1b). For the angiosperms, the relative proportion of $A A$ vs. $a a$ and $A a$ vs. $a a$ competitions will be determined by the outcrossing rate, and so the outcome of the model will vary with the mating system (Table 1 ).

The purging of recessive mutations is most effective when there is high variance in fitness among selfed zygotes. Lande et al. (1994) have demonstrated that when load is high, viability of selfed zygotes is uniformly near zero, and thus there is little opportunity for purging. Essentially, with high load, selfed seeds are inviable, and embryo competition will have little effect on the frequency of recessive mutations. Yet competition may occur between outcrossed embryos, or between outcrossed and biparentally inbred embryos. Biparentally inbred seeds do express inbreeding depression (Heywood, 1993), but are less inbred such that the inbreeding depression is less severe and some are likely to be viable.

Fostering embryo competition may allow the maternal plant selectively to eliminate deleterious mutations, without incurring the cost of provisioning a seed. However, such a mechanism is advantageous in the long term only if the bulk of mutations are expressed in the embryo, otherwise load expressed in the adult stage is protected from selection, and will accumulate. Because selective seed abortion is advantageous in the short term, one would expect selective seed abortion to be favoured by selection in natural populations, regardless of its long-term consequences. However, two other factors will influence the evolutionary advantage of enhancing embryo competition. First, alleles which increase the proportion of self-fertilized progeny enjoy a transmission 
advantage (Fisher, 1941). It is possible that by altering the relative numbers of selfed and outcrossed progeny, embryo competition negates this advantage. Secondly, if pollen limitation occurs frequently, the mutations accumulated in one generation are likely to be expressed the next, so that there is no advantage to enhancing embryo competition.

\section{Empirical evidence}

Currently, there is little evidence available that will distinguish among the various possible mechanisms of selective seed abortion. It is known that outcrossing rates increase as seeds develop, because of the elimination of inbred embryos, particularly where load is high (Sorensen, 1982). However, this does not allow discrimination among the models above, because all predict this finding (Table 1). Indeed, the increased outcrossing rate is consistent with the simple inviability of embryos expressing genetic load. Yet patterns of seed abortion across fruits (Herrara, 1990) indicate that seed death is not random, but rather, survival of one seed is often associated with the death of neighbouring seeds. This strongly suggests the action of some form of competition among developing embryos, and mitigates against the hypothesis that seed abortion simply reflects death of nonviable embryos. Caspar (1988) showed that allowing seed abortion to occur naturally in Cryptantha flava resulted in higher fitness of the progeny than manually aborting seeds at random. Thus naturally aborted seeds are viable, but have lower fitness than those embryos which develop, so that seed abortion increases mean fitness of progeny. However, all models of selective seed abortion predict increased fitness in the short term (the time span of most experiments) and hence, these experiments do not indicate the mechanism of embryo competition.

Discrimination among the models hinges upon whether the bulk of deleterious mutations are expressed in the embryo, and upon the characteristics of those mutations. Tanksley et al. (1981) demonstrated a high degree of overlap in gene expression of isozymes in sporophyte and gametophyte of Lycopersicon esculentum. This suggests that many load loci could be involved in selective seed abortion. Yet most isozymes are 'housekeeping genes' which are expected to be expressed in many cell types, and thus may overestimate the overlap of gene expression between embryo and adult sporophyte. The characteristics of loci expressed at different life stages may also differ. Outcrossing species express more inbreeding depression at early life stages than selfing species (Husband \& Schemske,
1995). This suggests that mutations with larger fitness effects act earlier in the life cycle of a plant than more mildly deleterious mutations, which seem to form the bulk of inbreeding depression at later life stages (Barrett \& Charlesworth, 1990; Latta \& Ritland, 1994).

Comparisons among related taxa practising different levels of embryo competition may discriminate among the potential mechanisms of competition. Among the Pinaceae, those species with fewer archegonia per seed are likely to experience fewer opportunities for embryo competition. These species also express greater inbreeding depression in the seedling to adult stages of their life cycle (R. M. Bush, pers. comm.). This pattern is consistent with selective abortion being based on the fitness of the zygotes (Fig. 1, Table 1), but not with the mating-type model. It thus suggests that most mutations causing load are expressed in both the developing embryo and the adult, and form the basis for competition among embryos within an ovule.

\section{Implications for management of domestic or ex situ stocks}

There is currently some interest in management schemes for captive stocks that would increase the level of embryo competition, such as supplemental mass-pollination (Daniels, 1978; Crook \& Friedman, 1992). By increasing the number of fertilizations, there is increased competition among embryos as well as an increased range of genotypes among which to select. Thus mean fitness of the progeny will be improved. However, a long-term perspective must be taken in order to assess the effectiveness of such a scheme. Embryo competition does not necessarily cause an increase in genetic load (Fig. 1), but it is not possible to determine the mechanism (and hence predict the long-term effects) of enhancing embryo competition from experiments which simply measure short-term fitness changes. It is especially important to note that even where selective abortion may increase the fitness of stocks over the long term, the accumulation of additional mutations can produce a dramatic loss of fitness if selective seed abortion is discontinued (Fig. 1). Thus, once a programme to enhance selective abortion is begun, it may be impossible to cease this programme without irreparable damage to the stocks. This is of particular concern for ex situ management of endangered species for conservation purposes, because often these stocks are already small and prone to the negative effects of inbreeding.

To predict the outcome of enhanced embryo com- 
petition over the long term requires that the mechanism of load and of competition be determined. This requires that the following predictions be tested. Embryo competition predicts not only that the amount of seed abortion will correlate with the genetic load of the maternal plant (as with inviability of selfed seeds: Karkainen \& Savolainen, 1992), but also that abortion will not be random among fruits (Herrera, 1990). If competition is based upon the mating-type, fitness will not increase with competition among embryos with the same mating-type. If such competition is instead mediated by the genotype of the embryo, then competition among seeds which are all selfed, or all outcrossed, will result in an increase of progeny fitness. Simple hand-pollination experiments where performance of the progeny is determined can provide much of this information, but it is important that these experiments go beyond the simple observation that embryo competition enhances progeny performance.

\section{Acknowledgements}

I would like to thank Ned Friedman for motivating this study, and for many useful discussions. Robin Bush and Brian Husband gave kind permission to cite unpublished results and made many helpful suggestions. This work was funded by a Postgraduate Scholarship from the Natural Sciences and Engineering Research Council of Canada.

\section{References}

BARRETT, s. C. H. AND CHARLESWORTH, D. 1991. Effects of a change in the level of inbreeding on the genetic load. Nature, 352, 522-524.

BAWA, K. S. AND WEBB, C. J. 1984. Flower, fruit and seed abortion in tropical forest trees: implications for the evolution of paternal and maternal reproductive patterns. Am. J. Bot., 71, 736-751.

CASPAR, B. 1988. Evidence for selective embryo abortion in Cryptantha flava. Am. Nat., 132, 318-326.

CHARLESWORTH, B., CHARLESWORTH, D. AND MORGAN, M. T. 1990. Genetic loads and estimates of mutation rates in highly inbred plant populations. Science, 347, $380-382$.

CHARLESWORTH, D., MORGAN, M. T. AND CHARLESWORTH, B. 1990. Inbreeding depression, genetic load and the evolution of outcrossing rates in a multilocus system with no linkage. Evolution, 44, 1469-1489.

CROOK, R. W. AND FRIEDMAN, W. F. 1992. Effects of pollen tube number and archegonium number on reproduction in Douglas-fir: significance for seed orchard management. Can. J. Forest Res., 22, 1483-1488.

DANIELS, J. D. 1978. Efficacy of supplemental mass pollina- tion in a Douglas-fir seed orchard. Silvae Genet., 27, 52-58.

FISHER, R. A. 1941. Average excess and average effect of a gene substitution. Ann. Eugen., 11, 53-63.

GORCHOV, D. L. AND ESTABRoOK, G. F. 1987. A test of several hypotheses for the determination of seed number in Amelanchier arborea, using simulated probability distributions to evaluate data. Am. J. Bot., 74, 1893-1897.

HAIG, D. 1992. Brood reduction in gymnosperms. In: Elgar, M. A. and Crespi, B. J. (eds) Cannibalism: Ecology and Evolution among Diverse Taxa, pp. 63-84. Oxford University Press, London.

HERRERA, C. M. 1990. Brood size reduction in Lavandula latifolia (Labiatae): A test of alternative hypotheses. Evol. Trends Plants, 4, 99-105.

HEYwOOD, J. S. 1993. Bi-parental inbreeding depression in the self-incompatible annual plant Gaillardia pulchella (Asteraceae). Am. J. Bot., 80, 545-550.

HOLTSFORD, T. P. AND ELLSTRAND, N. C. 1990. Inbreeding effects in populations of Clarkia tembloriensis (Onagraceae) with different natural outcrossing rates. Evolution, 44, 2031-2046.

HUSBAND, B. AND SCHEMSKE, D. w. 1995. Evolution of the magnitude and timing of inbreeding depression in plants. Evolution, (in press).

KARKAINEN, K. AND SAVOLAINEN, O. 1994. The degree of early inbreeding depression determines the selfing rate at the seed stage: model and results from Pinus sylvestris (Scots pine). Heredity, 71, 160-166.

KLEKOWSKI, E. J. 1982. Genetic load and soft selection in ferns. Heredity, 49, 191-197.

Kondrashov, A. s. 1985. Deleterious mutations as an evolutionary factor. II. Facultative apomixis and selfing. Genetics, 111, 635-653.

LANDE, R. AND SCHEMSKE, D. W. 1985. The evolution of self-fertilization and inbreeding depression in plants. I. Genetic models. Evolution, 39, 24-40.

LANDE, R., SCHEMSKE, D. W. AND SCHultz, s. T. 1994. High inbreeding depression, selective interference among loci and the threshold selfing rate for purging recessive lethal mutations. Evolution, 48, 965-978.

LATTA, R. G. AND RITLAND, K. 1994. The relationship between inbreeding depression and prior inbreeding among populations of four Mimulus taxa. Evolution, 48, 806-817.

S1MMONS, M. J. AND CROW, J. F. 1977. Mutations affecting fitness in Drosophila populations. Ann. Rev. Genet., 11, 49-78.

SORENSEN, F. C. 1982. The roles of polyembryony and embryo viability in the genetic system of conifers. Evolution, 36, 725-733.

TANKSLEY, S. D., ZAMIR, D. AND RICK, C. M. 1981. Evidence for extensive overlap of sporophytic and gametophytic gene expression in Lycopersicon esculentum. Science, 213, 453-455.

WIENS, D. 1984. Ovule survivorship, brood size, life history, breeding systems and reproductive success in plants. Oecologia, 64, 47-53. 